\title{
Angiotensin II upregulated the expression of microRNA-224 but not microRNA-21 in adult rat cardiac fibroblasts
}

\author{
QILAN NING and XIAOYING JIANG \\ Department of Genetics and Molecular Biology, School of Medicine, Xi'an Jiaotong University, \\ Xi'an, Shaanxi 710061, P.R. China
}

Received May 22, 2013; Accepted July 05, 2013

DOI: $10.3892 /$ br.2013.144

\begin{abstract}
The role of microRNA-21 (miRNA-21, miR-21) in cardiac fibrosis remains controversial, while the role of microRNA-224 (miRNA-224, miR-224) in cardiac fibroblasts has not been reported. Angiotensin II (Ang II) is known to play a pivotal role in the pathogenesis of cardiac fibrosis. The aim of this study was to confirm whether the expression of miR-21 and miR-224 is regulated by Ang II in adult rat cardiac fibroblasts. Semi-quantitative reverse transcription-polymerase chain reaction (RT-PCR) and quantitative PCR (qPCR) were performed to measure the levels of miR-21 and miR-224 in Ang II-treated or untreated adult rat cardiac fibroblasts. The RT-PCR, qPCR and previous miRNA array results demonstrated that treatment with Ang II (100 nM) for $24 \mathrm{~h}$ did not induce the increase of miR-21 in cardiac fibroblasts, although the level of miR-21 in cardiac fibroblasts was not considered as low. The results of the present study also demonstrated that Ang II significantly upregulated the expression of miR-224 in adult rat cardiac fibroblasts. Bioinformatic analysis revealed that the potential target genes of miR-224 included SMAD4, SMAD5, cyclin-dependent kinase 9 and early growth response $1 / 2$. In previous studies, it was reported that miR-224 was upregulated in tumors by promoting cell proliferation and targeting SMAD4. Those results indicated the potential roles of miR-224 in cardiac fibroblasts and cardiac fibrosis. In conclusion, results of the present study demonstrated that miR-21 was not induced by Ang II, whereas Ang II upregulated miR-224 expression in adult rat cardiac fibroblasts, a finding that may provide a starting point for the investigation of the potential role of miR-224 in cardiac fibrosis.
\end{abstract}

Correspondence to: Dr Xiaoying Jiang, Department of Genetics and Molecular Biology, School of Medicine, Xi'an Jiaotong University, 76 Yanta West Road, Xi'an, Shaanxi 710061, P.R. China E-mail: jiangxy@mail.xjtu.edu.cn

Key words: angiotensin II, cardiac fibroblasts, microRNA-224, microRNA-21

\section{Introduction}

Cardiac fibroblasts play a pivotal role in the development of cardiac fibrosis, which is closely associated with numerous cardiovascular diseases, including hypertension, myocardial infarction and cardiomyopathy $(1,2)$. Angiotensin II (Ang II) is considered to be a major factor in the pathogenesis of cardiac fibrosis $(3,4)$. Ang II has been shown to induce cardiac fibrosis by stimulating the proliferation of cardiac fibroblasts and promoting the aberrant deposition of extracellular matrix in the myocardial interstitium (5-7). It is well known that the majority of the actions of Ang II are mainly mediated via angiotensin II receptor type 1 (AGTR1) (8). Currently, AGTR1 blockers such as Losartan are widely used in the clinical setting for various cardiovascular diseases (8). However, the molecular mechanism underlying the effects of Ang II on cardiac fibroblasts requires elucidation by further studies.

MicroRNAs (miRNAs) are an endogenous conserved class of small non-coding RNAs of 18-25 nucleotides, which are generally considered to downregulate the expression of target genes at the post-transcriptional level $(9,10)$. Several miRNAs have been reported to be involved in cardiac fibrosis (11-17). Thum et al (11) reported that miRNA-21 (miR-21) contributed to cardiac fibrosis by targeting transcript Sprouty1 which inhibits mitogen-activated protein kinase signaling in cardiac fibroblasts. Roy et al (12) reported that miR-21 promoted cardiac fibrosis by targeting the phosphatase and tensin homologue which regulates the expression of matrix metalloproteinase 2 (MMP2). However, Patrick et al (18) observed that the genetic deletion of miR-21 did not affect the response of the heart to pressure overload or other type of stress and concluded that miR-21 was not essential for cardiac fibrosis. Liang et al (19) recently reported that the overexpression of miR-21 in cardiac fibroblasts reduced transforming growth factor- $\beta$ (TGF $\beta$ ) RIII expression and increased collagen content. Therefore, further studies are required to elucidate the role of miR-21 in cardiac fibroblasts.

Our previous array study demonstrated that 33 miRNAs were differentially expressed in cardiac fibroblasts in response to treatment with Ang II (100 $\mathrm{nM})$ for $24 \mathrm{~h}$, including miRNA-224 (miR-224) (20). The increase of miR-132, miR-125b-3p and miR-146b and the decrease of miR-300-5p, miR-204* and miR-181b expression has been confirmed by quantitative polymerase chain reaction (qPCR) (20). 
However, the level of miR-224 has not been determined in cardiac fibroblasts. In order to elucidate the effect of Ang II on the expression of miR-21 and miR-224, we measured the levels of the two miRNAs with semi-quantitative reverse transcription-polymerase chain reaction (RT-PCR) and qPCR in adult rat cardiac fibroblasts. Our results demonstrated that miR-21 was not induced by Ang II, whereas the expression of miR-224 was upregulated in Ang II-treated adult rat cardiac fibroblasts. Furthermore, bioinformatic analysis revealed that the potential target genes of miR-224 included SMAD4, SMAD5, cyclin-dependent kinase 9 (CDK9) and early growth response 1/2 (EGR1/2), which indicated the potential role of miR-224 in cardiac fibroblasts.

\section{Materials and methods}

Materials and animals. Collagenase, trypsin and Ang II were obtained from Sigma (St. Louis, MO, USA). Dulbecco's modified Eagle's medium (DMEM) and TRIzol reagent were obtained from Invitrogen Life Technologies (Carlsbad, CA, USA). The First Strand cDNA Synthesis kit was purchased from Fermentas (Burlington, ON, Canada). SYBR Premix Ex $\mathrm{Taq}^{\mathrm{TM}}$ II was purchased from Takara Bio, Inc. (Shiga, Japan). Sprague-Dawley (SD) rats were supplied from the Experimental Animal Center of Xi'an Jiaotong University. The animal experiments were approved by the University Committee of Laboratory Animal Care and Use and were performed according to the guidelines of the National Animal Research Center.

Isolation and culture of cardiac fibroblasts. Cardiac ventricular fibroblasts were obtained from the hearts of adult male SD rats weighing 250-300 g, as previously described (20). In brief, following rapid excision of the hearts, the fibroblasts were prepared by enzymatic digestion with a collagenase/trypsin solution. After a 2 -h period of attachment to uncoated culture plates, the cells that were weakly attached or unattached were rinsed free and discarded and the attached cells (mainly fibroblasts) were washed and grown in DMEM supplemented with $10 \%$ fetal bovine serum. The cardiac fibroblasts (passages 3-5) were grown to $80-90 \%$ confluence and serum-starved for $24 \mathrm{~h}$ prior to treatment.

Preparation of RNA. Following a 24-h serum starvation, adult rat cardiac fibroblasts were treated with Ang II (100 nM) for $24 \mathrm{~h}$. The cells were then harvested for RNA extraction using TRIzol reagent as previously described and RNAs were dissolved in RNase-free water (20). The RNA quantity was spectrophotometrically determined as A260 and A260/A280 ratio using the NanoDrop 1000 spectrophotometer (Thermo Fisher Scientific, Wilmington, DE, USA) and RNA quality was assessed by electrophoresis on a $1.2 \%$ agarose/formaldehyde gel. Isolated RNA was stored at $-70^{\circ} \mathrm{C}$ for subsequent quantitative analysis.

Semi-quantitative RT-PCR analysis of $m i R-21$. To measure the level of miR-21 in adult rat cardiac fibroblasts and investigate the effect of Ang II on the expression of miR-21, semi-quantitative RT-PCR was first used to measure the level of miR-21. Briefly, total RNAs from six pairs of control and Ang II-treated cardiac fibroblasts were extracted using TRIzol reagent. Complementary DNAs (cDNAs) were synthesized from total RNAs using the First Strand cDNA Synthesis kit with miRNA-specific primers (Table I). The $20-\mu 1$ reactions were incubated for $60 \mathrm{~min}$ at $42^{\circ} \mathrm{C}$ and for $5 \mathrm{~min}$ at $70^{\circ} \mathrm{C}$ and then stored at $-20^{\circ} \mathrm{C}$. Conventional PCR reactions were performed at $95^{\circ} \mathrm{C}$ for $10 \mathrm{~min}$, followed by 40 cycles at $95^{\circ} \mathrm{C}$ for $15 \mathrm{sec}$ and at $60^{\circ} \mathrm{C}$ for $30 \mathrm{sec}$. The PCR products were submitted to electrophoresis using a $2 \%$ agarose gel. U6 RNA was used as internal control to normalize the level of miR-21 by densitometry using the ImageJ 1.37 freeware for Windows. The optical densitometry results were expressed as means \pm standard error of mean (SEM).

$q P C R$ analysis of $m i R-21$ and $m i R-224$. To validate the level of miR-21 and confirm the level of miR-224 in adult rat cardiac fibroblasts, we performed stem-loop qPCR to quantify the levels of the two miRNAs. Briefly, total RNAs from six pairs of control and Ang II-treated cardiac fibroblasts were extracted using TRIzol reagent. cDNAs were synthesized from total RNAs using the First-Strand cDNA Synthesis kit with miRNA-specific primers, as mentioned above (Table I). qPCR was performed using SYBR Premix Ex Taq II in an iQ5 real-time PCR detection system (Bio-Rad, Hercules, CA, USA). The PCR reactions were performed at $95^{\circ} \mathrm{C}$ for $10 \mathrm{~min}$, followed by 40 cycles at $95^{\circ} \mathrm{C}$ for $15 \mathrm{sec}$ and at $60^{\circ} \mathrm{C}$ for $30 \mathrm{sec}$. The specificity of the PCR products was assessed by the melting curve analysis. U6 was used as an internal control to normalize miRNA. Relative quantitation of miR-21 and miR-224 expression was evaluated by the $2^{-\Delta \Delta \mathrm{Ct}}$ method.

Bioinformatic analysis and target prediction. Four online software programs were used for bioinformatic analysis and target prediction of miRNAs: TargetScan (http://www.targetscan. org), microRNA.org (http://www.microrna.org/microrna/ home.do), miRDB (http://mirdb.org/miRDB) and MicroCosm Targets (http://www.ebi.ac.uk/enright-srv/microcosm/htdocs/ targets/v5/).

Statistical analysis. Data are presented as means \pm SEM. The Student's t-test was used to compare data between the two groups. $\mathrm{P}<0.05$ was considered to indicate a statistically significant difference.

\section{Results}

Effect of Ang II on miR-21 level in adult rat cardiac fibroblasts. To investigate the effect of Ang II on the expression of miR-21 in adult rat cardiac fibroblasts, semi-quantitative RT-PCR was first used to measure the level of miR-21. Although the level of miR-21 was not very low compared to U6, stimulation with Ang II did not induce a significant increase of miR-21 in cardiac fibroblasts (Fig. 1). Moreover, our previous miRNA array analysis also demonstrated that Ang II increased the expression of miR-21 only by 1.056 -fold in adult rat cardiac fibroblasts (20). Furthermore, qPCR demonstrated that Ang II did not affect the level of miR-21 in adult rat cardiac fibroblasts (Fig. 2).

Effect of Ang II on miR-224 level in adult rat cardiac fibroblasts. Our previous miRNA array study (20) demonstrated that Ang II increased the expression of miR-21 by 2.09 -fold in adult rat cardiac fibroblasts. To confirm the change of 
Table I. miRNA primer sequences.

\begin{tabular}{|c|c|}
\hline miRNA & Sequences \\
\hline miR-21 & $\begin{array}{c}\text { RT primer: } \quad \text { '-GTCGTATCCAGTGCAGGGTCCGAGGTATTCGCACTGGATACGACTCAACA-3' } \\
\text { PCR primer F: 5'-CGGCTAGCTTATCAGACTGA-3' } \\
\text { R: 5'-GTGCAGGGTCCGAGGT-3' }\end{array}$ \\
\hline miR-224 & $\begin{array}{cc}\text { RT primer: } & \text { 5'-GTCGTATCCAGTGCAGGGTCCGAGGTATTCGCACTGGATACGACAAACGG-3' } \\
\text { PCR primer F: 5'-CGGCCAAGTCACTAGTGGTT-3' } \\
\text { R: 5'-GTGCAGGGTCCGA GGT-3' }\end{array}$ \\
\hline U6 & $\begin{array}{l}\text { PCR primer F: 5'-CTCGCTTCGGCAGCACA-3' } \\
\text { R: 5'-AACGCTTCACGAATTTGCGT-3' }\end{array}$ \\
\hline
\end{tabular}

RT, reverse transcription; PCR, polymerase chain reaction; F, forward; R, reverse.

A

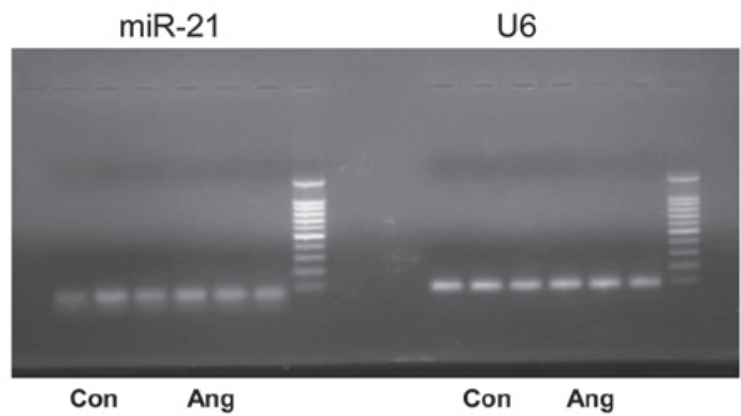

B

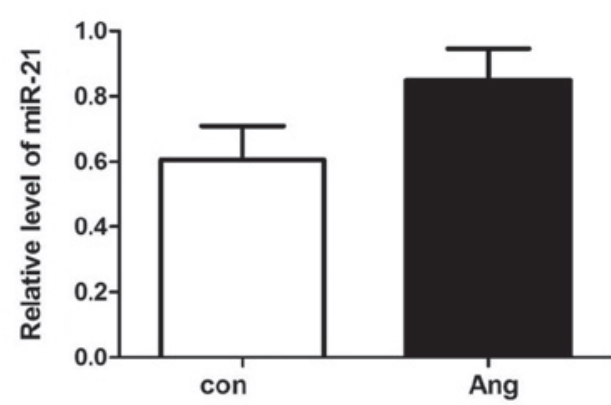

Figure 1. Semi-quantitative analysis of the effect of angiotensin II (Ang II) on miR-21 level in adult rat cardiac fibroblasts. Cardiac fibroblasts were treated with Ang II (100 nM) for $24 \mathrm{~h}$ and total RNAs were extracted. The level of miR-21 was measured with semi-quantitative reverse transcription-polymerase chain reaction. (A) Representative results of electrophoresis. (B) Quantitative analysis of miR-21 level relative to U6. Data are expressed as means \pm SEM ( $=6$ ). ${ }^{*} \mathrm{P}<0.05$ compared to the non-treatment group. Con, control; SEM, standard error of the mean.
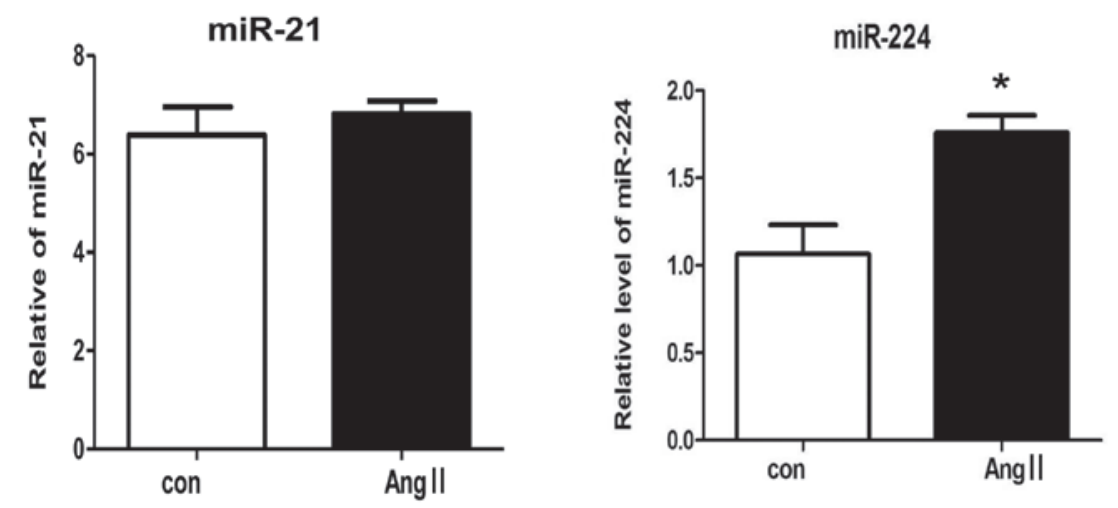

Figure 2. Quantitative polymerase chain reaction (qPCR) analysis of miR-21 and miR-224. The expression levels of RNA-miR-21 and RNA-miR-224 in cardiac fibroblasts treated by angiotensin II (Ang II) and untreated cells were measured by qPCR. Expression of miRNAs was normalized to endogenous U6 expression. Data are expressed as means \pm SEM $(n=6) .{ }^{*} \mathrm{P}<0.05$ compared to the non-treatment group. Con, control; SEM, standard error of the mean.

miR-224, qPCR was used to reassess the level of miR-224 in adult rat cardiac fibroblast treated by Ang II (100 nM) for $24 \mathrm{~h}$. The qPCR analysis revealed that Ang II significantly induced miR-224 expression in adult rat cardiac fibroblasts (Fig. 2). The upregulation of miR-224 induced by Ang II was verified by miRNA arrays and qPCR.

Bioinformatic analysis and target prediction of miR-224. After verifying that Ang II was able to induce a significant increase of miR-224 expression in adult rat cardiac fibroblasts, the potential target genes of miR-224 were analyzed using the online software programs TargetScan, microRNA.org, miRDB and MicroCosm Targets. The TargetScan analysis revealed that the potential target genes of miR-224 include SMAD4, H3 histone, family 3B (H3F3B), EGR2, SMAD5 and CDK9. The microRNA.org revealed that the potential target genes of miR-224 include EGR1, EGR2, H3F3B, SMAD4, SMAD5, CDK9, a disintegrin and metallopeptidase 
Table II. Potential target genes of miR-224.

\begin{tabular}{ll} 
miR-224 & \multicolumn{1}{c}{ Potential targets } \\
\hline TargetScan & SMAD4, H3F3B, EGR2, SMAD5, CDK9 \\
microRNA.org & EGR1, EGR2, H3F3B, SMAD4, SMAD5, CDK9, ADAM17, AGTR2, AGTR1a \\
miRDB & H3F3B, ADAM17, EGR2, API5, PIK3C2A \\
MicroCosm Targets & CDK9, MAPK8, ADAM33 \\
\hline
\end{tabular}

H3F3B, H3 histone, family 3B; EGR, early growth response; CDK9, cyclin-dependent kinase 9; ADAM, a disintegrin and metallopeptidase; AGTR, angiotensin II receptor; API5, apoptosis inhibitor 5; PIK3C2A, phosphoinositide-3-kinase, class 2, $\alpha$ polypeptide; MAPK, mitogen-activated protein kinase.

Table III. Reported function of miR-224.

\begin{tabular}{|c|c|c|c|c|}
\hline $\operatorname{miR}-224$ & Cell & Target genes & Function & Refs. \\
\hline Up & Hepatocellular carcinoma & API5 & Cell apoptosis and proliferation & 21,22 \\
\hline Up & Granulosa cell & SMAD4 & Cell proliferation & 23,24 \\
\hline Up & HepG2 cells & PAK4, MMP9 & Cell proliferation, migration and invasion & 25 \\
\hline Up & Human breast cancer cells & RKIP & Cell invasion and metastasis & 26 \\
\hline Up & Hepatocellular carcinoma & $\begin{array}{l}\text { CDC42, CDH1, PAK2, } \\
\text { BCL2, MAPK1 }\end{array}$ & $\begin{array}{l}\text { Cell proliferation, migration, invasion } \\
\text { and anti-apoptosis }\end{array}$ & 27 \\
\hline Up & Cancer associated with IBD & $\mathrm{p} 21$ & G1-S checkpoint & 28 \\
\hline
\end{tabular}

API5, apoptosis inhibitor 5; MMP9, matrix metalloproteinase 9; RKIP, Raf kinase inhibitor protein; BCL2, B-cell lymphoma 2; IBD, inflammatory bowel disease; PAK, p21-activating kinase; CDC, cell division control protein; MAPK, mitogen-activated protein kinase; Up, upregulated.

domain 17 (ADAM17), angiotensin II receptor type 2(AGTR2) and angiotensin II receptor type la (AGTR1a). The miRDB analysis revealed that apoptosis inhibitor 5 (API5), phosphoinositide-3-kinase, class 2, $\alpha$ polypeptide (PIK3C2A), $\mathrm{H} 3 \mathrm{~F} 3 \mathrm{~B}, \mathrm{ADAM} 17$ and EGR2 are the potential target genes of miR-224. MicroCosm Targets analysis also indicated that CDK9, MAPK8 and ADAM33 are the potential target genes of miR-224 (Table II).

\section{Discussion}

Several studies demonstrated that miR-21 is involved in the process of cardiac fibrosis by regulating the expression of Sprouty1, MMP2 and TGF $\beta$ RIII $(11,12,19)$. However, evidence presented by other studies does not support the role of miR-21 in cardiac fibrosis. Patrick et al (18) reported that genetic deletion of miR-21 did not alter the response of the heart to pressure overload or other type of stress and concluded that miR-21 was not essential for cardiac fibrosis. Our semi-quantitative RT-PCR, miRNA arrays and qPCR studies demonstrated that treatment with Ang II (100 nM) for $24 \mathrm{~h}$ did not induce the increase of miR-21 expression in cardiac fibroblasts, although the level of miR-21 in cardiac fibroblasts was not considered to be low. Therefore, further investigations are required to elucidate the role of miR-21 in cardiac fibroblasts.

Our previous miRNA array studies and the qPCR analysis demonstrated that Ang II significantly induced the increase of miR-224 expression in adult rat cardiac fibroblasts (Fig. 2). Furthermore, bioinformatic analysis revealed that the potential target genes of miR-224 included SMAD4, SMAD5, CDK9, API5, EGR1/2, H3F3B, AGTR2 and AGTR1a, which indicated the potential role of miR-224 in cardiac fibroblasts. It is well known that SMAD molecules are distinctly associated with the TGF $\beta$ pathway and fibrosis, whereas CDK9, API5 and EGR1/2 are associated with cell proliferation. If AGTR2 and AGTR1a are indeed target genes of miR-224, miR-224 may inhibit the action of Ang II. However, this hypothesis requires confirmation by further studies.

Several studies have confirmed potential target genes, thus predicting the function of miR-224 (Table III): Wang et al (21) reported that miR-224 was significantly upregulated in hepatocellular carcinoma and affected cell apoptosis and proliferation through targeting API5; Wang et al (22) reported that a high miR-224 expression in hepatocellular carcinoma was regulated by histone deacetylase 1, histone deacetylase 3 and E1A binding protein p300; Yao et al (23) reported that miR-224 promoted TGF $\beta 1$-induced granulosa cell proliferation by targeting SMAD4; Liang et al (24) demonstrated that p53/p65 was able to inhibit miR-224 expression in granulosa cells; Li et al (25) observed that miR-224 was upregulated in HepG2 cells and was involved in cell proliferation, migration and invasion; Huang et al (26) reported that miR-224 was significantly upregulated in breast cancer cell lines and regulated cell invasion and tumor metastasis by targeting 
Raf kinase inhibitory protein; Zhang et al (27) reported that miR-224 in hepatocellular carcinoma affected cell proliferation, migration and exerted an anti-apoptotic effect; and Olaru et al (28) reported that miR-224 was highly expressed in cancers associated with inflammatory bowel disease by targeting p21.

The literature mentioned above demonstrated that miR-224 regulates cell proliferation in tumor cells. In our study, Ang II significantly upregulated the expression of miR-224 in adult rat cardiac fibroblasts. Ang II has been shown to stimulate the proliferation of cardiac fibroblasts, which is an important factor in cardiac fibrosis (5-7). Therefore, we hypothesized that miR-224 may also be involved in the process of cardiac fibroblast proliferation and cardiac fibrosis. The present study may provide a starting point to investigate the target genes of miR-224 and elucidate its role in cardiac fibrosis.

\section{Acknowledgements}

This study was supported by the Fundamental Research Funds for the Central Universities (no. 08143023) and grants from the National Science Foundation of China (no. 31100834).

\section{References}

1. Takeda N, Manabe I, Uchino Y, Eguchi K, Matsumoto S, Nishimura S, Shindo T, Sano M, Otsu K, Snider P, Conway SJ and Nagai R: Cardiac fibroblasts are essential for the adaptive response of the murine heart to pressure overload. J Clin Invest 120: 254-265, 2010.

2. Porter KE and Turner NA: Cardiac fibroblasts: at the heart of myocardial remodeling. Pharmacol Ther 123: 255-278, 2009

3. Ren J, Yang M, Qi G, Zheng J, Jia L, Cheng J, Tian C, Li H, Lin X and Du J: Proinflammatory protein CARD9 is essential for infiltration of monocytic fibroblast precursors and cardiac fibrosis caused by Angiotensin II infusion. Am J Hypertens 24: 701-707, 2011.

4. Huang XR, Chung AC, Yang F, Yue W, Deng C, Lau CP, Tse HF and Lan HY: Smad3 mediates cardiac inflammation and fibrosis in angiotensin II-induced hypertensive cardiac remodeling. Hypertension 55: 1165-1171, 2010.

5. Olson ER, Shamhart PE, Naugle JE and Meszaros JG: Angiotensin II-induced extracellular signal-regulated kinase 1/2 activation is mediated by protein kinase Cdelta and intracellular calcium in adult rat cardiac fibroblasts. Hypertension 51: 704-711, 2008

6. Schellings MW, Vanhoutte D, van Almen GC, Swinnen M, Leenders JJ, Kubben N, van Leeuwen RE, Hofstra L, Heymans S and Pinto YM: Syndecan-1 amplifies angiotensin II-induced cardiac fibrosis. Hypertension 55: 249-256, 2010.

7. Lijnen PJ, Van Pelt JF and Fagard RH: Stimulation of reactive oxygen species and collagen synthesis by angiotensin II in cardiac fibroblasts. Cardiovasc Ther 30: e1-e8, 2012.

8. Zhang P, Su J, King ME, Maldonado AE, Park C and Mende U: Regulator of $\mathrm{G}$ protein signaling 2 is a functionally important negative regulator of angiotensin II-induced cardiac fibroblast responses. Am J Physiol Heart Circ Physiol 301: H147-H156, 2011.

9. Jiang X, Tsitsiou E, Herrick SE and Lindsay MA: MicroRNAs and the regulation of fibrosis. FEBS J 277: 2015-2021, 2010.

10. Diez J: Do microRNAs regulate myocardial fibrosis? Nat Clin Pract Cardiovasc Med 6: 88-89, 2009.

11. Thum T, Gross C, Fiedler J, Fischer T, Kissler S, Bussen M, Galuppo P, Just S, Rottbauer W, Frantz S, et al: MicroRNA-21 contributes to myocardial disease by stimulating MAP kinase signalling in fibroblasts. Nature 456: 980-984, 2008.
12. Roy S, Khanna S, Hussain SR, Biswas S, Azad A, Rink C, Gnyawali S, Shilo S, Nuovo GJ and Sen CK: MicroRNA expression in response to murine myocardial infarction: miR-21 regulates fibroblast metalloprotease- 2 via phosphatase and tensin homologue. Cardiovasc Res 82: 21-29, 2009.

13. van Rooij E, Sutherland LB, Thatcher JE, DiMaio JM, Naseem RH, Marshall WS, Hill JA and Olson EN: Dysregulation of microRNAs after myocardial infarction reveals a role of miR-29 in cardiac fibrosis. Proc Natl Acad Sci USA 105: 13027-13032, 2008.

14. Duisters RF, Tijsen AJ, Schroen B, et al: miR-133 and miR-30 regulate connective tissue growth factor: implications for a role of microRNAs in myocardial matrix remodeling. Circ Res 104: 170-178, 2009.

15. Shan H, Zhang Y, Lu Y, Zhang Y, Pan Z, Cai B, Wang N, Li X, Feng T, Hong Y and Yang B: Downregulation of miR-133 and miR-590 contributes to nicotine-induced atrial remodelling in canines. Cardiovasc Res 83: 465-472, 2009.

16. Limana F, Esposito G, D'Arcangelo D, Di Carlo A, Romani S, Melillo G, Mangoni A, Bertolami C, Pompilio G, Germani A and Capogrossi MC: HMGB1 attenuates cardiac remodelling in the failing heart via enhanced cardiac regeneration and miR-206-mediated inhibition of TIMP-3. PLoS One 6: e19845, 2011.

17. Wang J, Huang W, Xu R, Nie Y, Cao X, Meng J, Xu X, Hu S and Zheng Z: MicroRNA-24 regulates cardiac fibrosis after myocardial infarction. J Cell Mol Med 16: 2150-2160, 2012.

18. Patrick DM, Montgomery RL, Qi X, Obad S, Kauppinen S, Hill JA, et al: Stress-dependent cardiac remodeling occurs in the absence of microRNA-21 in mice. J Clin Invest 120: 3912-3916, 2010.

19. Liang H, Zhang C, Ban T, Liu Y, Mei L, Piao X, Zhao D, Lu Y, Chu W and Yang B: A novel reciprocal loop between microRNA-21 and TGF $\beta$ RIII is involved in cardiac fibrosis. Int J Biochem Cell Biol 44: 2152-2160, 2012.

20. Jiang X, Ning Q and Wang J: Angiotensin II induced differentially expressed microRNAs in adult rat cardiac fibroblasts. J Physiol Sci 63: 31-38, 2013.

21. Wang Y, Lee AT, Ma JZ, Wang J, Ren J, Yang Y, Tantoso E, Li KB, Ooi LL, Tan P and Lee CG: Profiling microRNA expression in hepatocellular carcinoma reveals microRNA-224 up-regulation and apoptosis inhibitor-5 as a microRNA-224-specific target. J Biol Chem 283: 13205-13215, 2008.

22. Wang Y, Toh HC, Chow P, Chung AY, Meyers DJ, Cole PA, Ooi LL and Lee CG: MicroRNA-224 is up-regulated in hepatocellular carcinoma through epigenetic mechanisms. FASEB J 26: 3032-3041, 2012.

23. YaoG,YinM,Lian J,Tian H,Liu L,LiX and Sun F: MicroRNA-224 is involved in transforming growth factor- $\beta$-mediated mouse granulosa cell proliferation and granulosa cell function by targeting Smad4. Mol Endocrinol 24: 540-551, 2010.

24. Liang M, Yao G, Yin M, Lu M, Tian H, Liu L, Lian J, Huang X and Sun F: Transcriptional cooperation between $\mathrm{p} 53$ and NF- $\mathrm{KB}$ p65 regulates microRNA-224 transcription in mouse ovarian granulosa cells. Mol Cell Endocrinol 370: 119-129, 2013.

25. Li Q, Wang G, Shan JL, Yang ZX, Wang HZ, Feng J, Zhen JJ, Chen C, Zhang ZM, Xu W, Luo XZ and Wang D: MicroRNA-224 is upregulated in HepG2 cells and involved in cellular migration and invasion. J Gastroenterol Hepatol 25: 164-171, 2010.

26. Huang L, Dai T, Lin X, Zhao X, Chen X, Wang C, Li X, Shen H and Wang X: MicroRNA-224 targets RKIP to control cell invasion and expression of metastasis genes in human breast cancer cells. Biochem Biophys Res Commun 425: 127-133, 2012.

27. Zhang Y, Takahashi S, Tasaka A, Yoshima T, Ochi H and Chayama K: Involvement of microRNA-224 in cell proliferation, migration, invasion, and anti-apoptosis in hepatocellular carcinoma. J Gastroenterol Hepatol 28: 565-575, 2013.

28. Olaru AV, Yamanaka S, Vazquez C, Mori Y, Cheng Y, Abraham JM, Bayless TM, Harpaz N, Selaru FM and Meltzer SJ: MicroRNA-224 negatively regulates p21 expression during late neoplastic progression in inflammatory bowel disease. Inflamm Bowel Dis 19: 471-480, 2013 